This item was submitted to Loughborough's Research Repository by the author.

Items in Figshare are protected by copyright, with all rights reserved, unless otherwise indicated.

\title{
Field assisted sintering of nanostructured zirconia-alumina ceramics for demanding applications
}

PLEASE CITE THE PUBLISHED VERSION

http://dx.doi.org/10.1080/0371750X.2016.1172981

PUBLISHER

Taylor \& Francis

VERSION

AM (Accepted Manuscript)

\section{PUBLISHER STATEMENT}

This work is made available according to the conditions of the Creative Commons Attribution-NonCommercialNoDerivatives 4.0 International (CC BY-NC-ND 4.0) licence. Full details of this licence are available at: https://creativecommons.org/licenses/by-nc-nd/4.0/

\section{LICENCE}

CC BY-NC-ND 4.0

\section{REPOSITORY RECORD}

Downs, John A., Annapoorani Ketharam, and Bala Vaidhyanathan. 2016. "Field Assisted Sintering of Nanostructured Zirconia-alumina Ceramics for Demanding Applications". figshare. https://hdl.handle.net/2134/21146. 
Field assisted sintering of nanostructured zirconia-alumina

ceramics for demanding applications

4

John A. Downs

j.a.downs@lboro.ac.uk

Department of Materials

Loughborough University

Loughborough, United Kingdom

8

10

11

12

13

14

15

16

17

18

19

20

21

22

23

24

25

26 
Abstract:

2 Flash and Field Assisted Sintering Techniques (FAST) have been demonstrated in many 3 singular ceramic systems. However, little has been done to thoroughly investigate this phenomenon in binary systems. Zirconia-alumina composites are of interest because of their widespread use in demanding situations for health care, petro-chemical and energy applications. The prospect of minimising grain growth associated with FAST whilst achieving maximum densification is vital for the above applications to improve performance. Flash sintering behaviour of several zirconia-alumina formulations was investigated under a range of DC electric fields up to $700 \mathrm{~V} / \mathrm{cm}$. At low alumina contents ( $<10 \mathrm{wt} \%)$ the flash behaviour was controlled by the zirconia phase; at intermediate compositions (10 wt $\%$ - $70 \mathrm{wt} \%$ ) show a composite effect; and at high alumina contents (>75 wt\%) the effectiveness of field assisted sintering drops off. These pointers allowed to gain further insight into the mechanisms of flash sintering and will help to develop pathways for the adaptation of the technique to process complex ceramic systems.

Keywords: Flash Sintering, FAST, Zirconia-Toughened Alumina, zirconia, bioceramics 17

\section{Introduction}

Zirconia-toughened alumina (ZTA) composites are ideal candidate materials for demanding applications that require high hardness and high fracture toughness. Here, we are concerned with biomedical applications like total hip replacements (THR) and total knee replacements (TKR), but these results can be extended to energy applications like solid oxide fuel cell (SOFC) electrolytes and petro-chemical valve seats for oil drilling. This composite ceramic takes advantage of the high hardness and light weight of alumina and the high fracture toughness of yttria-stabilized zirconia (YSZ) resulting in a superior wear resistant material optimal for these applications. It is also more biocompatible than the corresponding metallic or polymeric components that can be toxic and produce wear debris upon degradation. Despite the apparent advantages of ZTA and also of the corresponding monolithic YSZ which are also used in biomedical applications, implants have been found to prematurely fail in-vivo after a couple years when the expected lifetime should exceed more than 10 years and preferably 20 years ${ }^{1-3}$. Upon closer examination, the YSZ component failure was found to occur due to subcritical crack propagation. Especially YSZ and ZTA 
1 subcritical crack growth is chemical corrosion due to hydrothermal aging (HTA) ${ }^{4,5}$ of the 2 zirconia phase.

The mechanisms for HTA still remain unclear. What is commonly accepted is that during high temperature sintering yttria preferentially segregates to the grain boundary resulting in concentration gradients of yttria across individual YSZ grains. Yttria is added to zirconia to stabilize the high temperature tetragonal phase ${ }^{6}$. This stabilization gives YSZ its fracture toughness because when fracture does occur, the meta-stable tetragonal phase transitions to the stable monoclinic phase. The energy required for this phase transformation is absorbed at the crack tip that slows down the crack propagation and is responsible for the high fracture toughness of YSZ and ZTA. When yttria segregation to the grain boundaries occurs, the cores of the grains are left under-stabilized If the core is penetrated by a crack tip, there is no longer the advantageous phase transformation and, hence, no increased fracture toughness. In wet environments, since crack tip corrosion is severe due to HTA and the advantages of the yttria stabilization are no longer present in large volumes of the material, crack propagation continues unhindered ${ }^{1,7}$. In implant applications where exposure to wet environments can be for $10-20$ years, these mechanisms must be considered. These issues are further exasperated in the steam sterilization condition used to treat implants and surgical tools before use. The sterilising temperatures have been found ideal for HTA and the high pressures increase the degradation resulting in accelerated aging conditions. Even after short treatment times, aging is noticed to occur resulting in increased surface roughness ${ }^{7}$ In SOFCs, HTA occurs during heating and cooling when the cells pass through the $200-300^{\circ} \mathrm{C}$ temperature range with wet fuels, and this results in the degradation of the YSZ electrolytes ${ }^{5}$.

Various methods have been shown to prevent HTA: ensuring high density, changing doping/stabilization levels, use of additional dopants, and various surface treatments to avoid the initial chemical attack and initial crack propagation ${ }^{8}$. Of importance here, is the reduction of grain size and grain size dependent phase-stabilisation ${ }^{9,10}$. By reducing grain size to 200$300 \mathrm{~nm}{ }^{11-13}$, HTA resistance begins to increase. If grain sizes are further reduced to an average of $90 \mathrm{~nm}$, 3YSZ has been shown to be completely immune to HTA, showing no tetragonal to monoclinic transformation even in the most aggressive steam environment and the equivalent of 1350 years in-vivo ${ }^{7}$. There was no detected yttria gradient across grains because of the nanometric grain size, meaning, there is no chemical driving force for HTA and the tetragonal-monoclinic transformation is prevented leaving hardness and fracture toughness values at pre-treatment levels. 
Control of grain growth has been a historic issue with the sintering of ceramics. In Ref

$2\left[{ }^{7}\right]$ the HTA resistant nano-YSZ was prepared by a two-step sintering procedure. In this case, 3 the sintering cycle was $6 \mathrm{sec}$ at $1150{ }^{\circ} \mathrm{C}$ and up to $10 \mathrm{hr}$ at $1050^{\circ} \mathrm{C}$ to achieve full density.

4 Two-step sintering, while effective, requires very long sintering time to achieve full densities and is therefore equipment and energy intensive due to the long times and high temperatures involved.

Field Assisted Sintering Techniques (FAST) are a class of sintering methodologies that use the application of various electric, magnetic and electromagnetic fields to accelerate sintering, reduced processing temperature, and shorten dwell times, resulting in fine grained material when compared to conventional or two-stage sintering. The most common FAST methodologies include techniques like Spark Plasma Sintering (SPS) ${ }^{14}$ and microwave assisted sintering as well as the more recently developed flash sintering (FS). SPS has very limited shape capability, a hindrance for biomedical needs, requires highly specialized, very expensive equipment that operates mainly in inert atmosphere (due to the use of graphite die), and requires additional post processing to deal with carbon contamination. Microwave assisted sintering, in pure (MW only) and hybrid heating (MW and radiant heating) forms, are more adapted to processing of complex shapes but also require specialized equipment (magnetron and containment) and the materials that can be used are limited. ZTA is an ideal candidate because zirconia is a good microwave absorber while alumina is microwave transparent. Fine grained ZTA ceramics have been produced by pure microwave of hybridmicrowave sintering in shorter times ${ }^{15,16}$. Flash sintering is a newly developed technique that uses the direct application of DC or AC currents to process ceramic materials. It has been found that below a threshold E-field strength, sintering has been found to be moderately accelerated with sintering onset occurring at similar temperatures as in conventional sintering. If the E-field strength exceeds this threshold, sintering onset temperatures are lowered significantly and sintering times reduced to the order of seconds rather than hours/days. The E-fields and current required, their relation to temperature and the threshold temperature for the onset of flash behaviour are material dependent and have relationships to their conductivity. The effect of flash sintering on grain growth has resulted in mixed observations. In some cases, grain growth is shown to be accelerated, even at the low processing temperatures and in some cases, grain growth is reduced or prevented. There appears to be a useful processing window (E-field strength-temperature- current density) that allow for good sintering while allowing for grain growth retardation. These regimes remain determined empirically for each material being examined. 
3YSZ was the first material used to demonstrate the flash sintering technique ${ }^{17}$. The critical E-field for the onset of flash sintering was found to be $\sim 50 \mathrm{~V} / \mathrm{cm}$. With $120 \mathrm{~V} / \mathrm{cm}$, sintering occurred to near full density at $850^{\circ} \mathrm{C}$ in a matter of seconds. Conventional treatments would be at $1450{ }^{\circ} \mathrm{C}$ for $2-5$ hours. Flash sintering in alumina has also been investigated with very different results ${ }^{17}$. With $120 \mathrm{~V} / \mathrm{cm}$, sintering occurred to near full density at $850^{\circ} \mathrm{C}$ in a matter of seconds. Convention treatments would b at $1450{ }^{\circ} \mathrm{C}$ for $2-5$ hours. In pure alumina, flash sintering was not observed up to E-field strengths of $1000 \mathrm{~V} / \mathrm{cm}$ 18 . With the addition of $0.25 \mathrm{wt} \% \mathrm{MgO}$, a typical liquid phase sintering aid, accelerated sintering occurred with the application of $500 \mathrm{~V} / \mathrm{cm}$ and flash sintering observed with 1000 $\mathrm{V} / \mathrm{cm}$. In the ZTA system, only 50:50 vol\% (60 wt\% 3YSZ) has been investigated and is the only biphasic investigation we are aware of ${ }^{19}$. It was found that the composite system shows a good flash sinterability, with the behaviour determined primarily by the zirconia phase.

However, the full range of zirconia-alumina ratios, especially in the biomedical range where ZTA is more useful is not investigated through FS. Flash sintering has significant potential for biomedical grade ZTA fabrication because more complex parts can be achieved compared to SPS and grain growth reduction is possible in the ZTA system, potentially eliminating the problems associated with HTA. Also, with flash sintering processing time and sintering temperatures can be reduced, thus minimising equipment demand and energy requirements.

The development of an effective flash sintering program is a methodical process. In flash sintering, the first thing that must be understood is the relationship between the applied Efield and its effect of the sintering temperature. Here the effect of various E-filed is investigated across a range of ZTA compositions starting with pure zirconia and increasing alumina content to the compositional range where structural ZTA applications are characterized (25ZTA) for biomedical needs. The electrical characterization is the first step towards having a basic understanding of the densification and microstructural effects of flash sintering on ZTA composites. After initial understanding of the electrical behaviour and the relationship between composition, E-field, and flash sintering temperature, more rigorous electrical controls can be implemented to produce well sintered materials. Further investigations will ensue to understand the effect of sintering treatments on grain size, HTA behaviour and mechanical properties. Such a process would have far reaching benefits in biomedical, petro-chemical and SOFC applications where HTA results in material failures and shortened lifetimes with fine grain sizes that can be exploited for HTA resistance in 


\section{Experimental:}

3 Nano-ZTA powders were prepared by spray freeze drying (SFD). SFD allows for a well 4 dispersed ZTA material with good flowability and good crushability of the produced agglomerates. First, nano-3YSZ (nominal grain size $30 \mathrm{~nm}$ ) and nano-alumina (nominal grain size $150 \mathrm{~nm}$ ) suspensions (MELox Chemicals) with concentration of $22.5 \mathrm{wt} \%$ and $43.7 \mathrm{wt} \%$, respectively, were mixed in the required amounts to achieve the desired zirconia/alumina weight ratios (Table I). Under constant stirring, the suspension $\mathrm{pH}$ was adjusted to 11 using tetra-methyl ammonium hydroxide (TMAH). This $\mathrm{pH}$ facilitates the effectiveness of the triammonium citrate (TAC) dispersant. Next, $3 \mathrm{wt} \%$, based on the ceramic solid content, TAC was slowly added. The dispersed suspension was then concentrated to $55 \mathrm{wt} \%$ by heating under constant stirring at $60^{\circ} \mathrm{C}$ until the correct volume was achieved. During concentration, the suspension was sonicated with a high energy sonication probe at regular intervals to break any agglomerates. Once the suspension reached the required concentration and was confirmed by drying a small sample, 3-methyl-2-butanol was added as a foaming agent. The suspension was sprayed using a Buchi Labortechnik AG twin-fluid atomiser into a liquid nitrogen bath at a rate of $3.8 \mathrm{l} / \mathrm{min}$. The resulting powder was freeze-dried dried for 48-72 hr (VirTis Benchtop BTK-2 Freeze Drier). Once dried, the powder was sieved keeping the fraction between 125 $\mu \mathrm{m}$ and $250 \mu \mathrm{m}$ for further processing.

\subsection{Sample Preparation}

The dried and sifted powders were uniaxially pressed in a $10 \mathrm{~mm}$ diameter die with $375 \mathrm{MPa}$ to produce disks of $\sim 3 \mathrm{~mm}$ thickness. To help homogenize stress gradients on the disk edges and minimize issues of delamination, samples were subsequently isopressed at 200 $\mathrm{MPa}$. After pressing, organics were removed by heating to $700^{\circ} \mathrm{C}$ for $2 \mathrm{hr}$ with heating and cooling rates of $2^{\circ} \mathrm{C} / \mathrm{min}$.

\subsection{Flash Sintering and Characterization}

For flash sintering, a thin layer of platinum paint was applied to both circular faces of the disk being measured and allowed to dry at room temperature. The disk was sandwiched between graphite sheet electrodes that were in contact with the platinum current carrying wires. The sample was heated in static air with no applied E-field to $450^{\circ} \mathrm{C}$ and held for at least 30 min to allow for temperature homogenization. After the soak, the voltage required to 
1 at a constant heating rate of $10^{\circ} \mathrm{C} / \mathrm{min}$ until a flash sintering event occurred as observed using

2 a high resolution CCD camera. E-fields of $300 \mathrm{~V} / \mathrm{cm}, 500 \mathrm{~V} / \mathrm{cm}$ or $700 \mathrm{~V} / \mathrm{cm}$ were applied to

3 allow for a wider examination of the zirconia/alumina compositional range, where alumina

4 rich materials would not sinter at the lower E-field strengths. Power supply current was

5 limited to 1 A to operate in the current limiting regime. Current and voltage were measured

6 by digital multimeters and the furnace temperature near the sample by a thermocouple

7 mounted within $3 \mathrm{~mm}$ of the sample in the holder. After sintering, fracture surfaces were

8 plated with gold/palladium and examined by FEG-SEM (JEOL JSM-7800F) to observe the

9 extent of sintering.

\section{Results and Discussion:}

The power dissipation during flash sintering behaviour for the range of compositions with a fixed E-field of $300 \mathrm{~V} / \mathrm{cm}$ is shown Figure 1. Power dissipation is given as volumetric dissipation $\mathrm{W} / \mathrm{mm}^{3}$ on a logarithmic scale against the inverse temperature. In the case of pure 3YSZ and high zirconia content 90ZTA samples, the power dissipation behaviour is very similar. In both compositions, with $300 \mathrm{~V} / \mathrm{cm}$ applied, electrical runaway and sintering occurred at $762^{\circ} \mathrm{C}$. Increasing the alumina content to $20 \mathrm{wt} \%$, the sintering behaviour begins to shift, and the sintering occurred at $\sim 20^{\circ} \mathrm{C}$ higher at $783^{\circ} \mathrm{C}$. At the alumina rich end of the composition, 30ZTA flash sintered at $1001^{\circ} \mathrm{C}$, but 25ZTA did not exhibit flash behaviour in the temperature regime investigated with $300 \mathrm{~V} / \mathrm{cm}$. The power dissipation does not continue to increase in this case and begins to taper-off above $1000{ }^{\circ} \mathrm{C}$. This is attributed to decomposition and oxidation of the graphite electrodes at these temperatures, the limiting factor in this experimental configuration.

As has been observed in other materials, the onset of flash sintering occurs at a threshold power dissipation that is independent of the composition. The transitions from activated type behaviour to runaway occurred at a critical power dissipation of $0.02 \mathrm{~W} / \mathrm{mm}^{3}$. Below this transition, all compositions exhibit linear behaviour. These results are for measurements performed under constant E-field, meaning any change in power dissipation results were due to changes in conductivity. The slopes for all compositions are similar indicating that no significant changes in conductivity activation energy occurred. The shifts to higher temperatures for a given power density and the corresponding shift in flash sintering temperature with increasing alumina content resulted from total changes in bulk conductivity as would be expected with increases in alumina content. 
The composition of 25ZTA is of interest because it is one of the common compositions for ZTA applications. The behaviour under three different E-fields is shown in 3 Figure 2. By increasing the E-field strength from $300 \mathrm{~V} / \mathrm{cm}$ to $500 \mathrm{~V} / \mathrm{cm}$, the change in conductivity at the higher E-field allows the onset of flash sintering at $1046^{\circ} \mathrm{C}$. With 700 $\mathrm{V} / \mathrm{cm}$, the flash sintering temperature decreased further to $920^{\circ} \mathrm{C}$. Unlike what was found with a fixed E-field strength and variation of zirconia content, the onset power for flash decreases with increasing E-field where the critical power density for $700 \mathrm{~V} / \mathrm{cm}$ is similar to the $0.02 \mathrm{~W} / \mathrm{mm}^{3}$ found before. For $500 \mathrm{~V} / \mathrm{cm}$, the transition to flash sintering is also not as clear. Again, these differences may be due to electrodes decomposition and require further investigation to see if the transition is a result of the electrode degradation or if the critical power dissipation is truly a function of E-field.

In this work, the emphasis was placed on finding the electrical behaviour of the material and the effect of composition. This is the first step in determining an effective flash sintering programme for any material. In these experiments, current limits were set high (2 A), and times in the current controlled regime after the onset current runaway were not closely controlled. Figure 3 shows two samples of 25 ZTA sintered at $700 \mathrm{~V} / \mathrm{cm}$. These are typical sintering results regardless of compositions, E-field or temperature. In both samples, currents localized at one point where the sample surface appears blackened and cratered. The temperatures that resulted from joule heating during current runaway also resulted in melting of the ceramic phases in these regions. The localizations of current can occur in any location on the sample. The final issue is the cracking of the sample. In most cases, when the sample is removed it is broken in two or more pieces. When this breaking occurs is not clear- it may occur during the sudden heating when flash sintering is initiated, during cooling once the power supply is switched off and the sample is allowed to cool, or from differential shrinkage of areas of different density. The effects of these localized events, however, do no effect the relationship between the applied E-field and the onset of flash behaviour. If experiments are stopped at or before the shoulder of the transition right before the runaway, no sintering and no localized zones were seen.

The different regions associated with different extent of sintering are also observed in the microstructure. Microstructures of the fracture surfaces taken from the samples discussed above are given in Figure 4. There are under-sintered regions (Fig 4a) which occur farthest from where flash sintering started-determined by the centre of the melted region-and closest to the edges where heat losses occur at a higher rate. In these areas, only neck 
1 alumina and smaller zirconia grains. Even though the extent of sintering is low, the fact

2 remains that sintering has occurred at furnace temperatures much lower than would be 3 expected by conventional methods. The majority of the sample is well sintered (Fig 4b).

4 Grains are well formed with no evident open porosity. Sintered grains are of a similar dimension of the under-sintered grains (Fig 4a) meaning no significant grain growth had occurred during the flash sintering. The sintered grain size is less than $500 \mathrm{~nm}$. Again, the alumina remains the larger grain and zirconia the smaller grain. This material is expected to be completely resistant to HTA because the YSZ grains are $<100 \mathrm{~nm}$. Finally, in the melted region (Fig 4c), there is a glued microstructure where there is evidence of melting with a dispersion of frozen grains in the melted matrix. A large amount of grain growth has occurred in these regions with the grain sizes of $\sim 10 \mu \mathrm{m}$.

Preventing the localization of sintering and melting to achieve a full density with a uniform microstructure is one of the most important and difficult tasks when flash sintering. With an understanding of the relationship between the onset of current runaway for a given ZTA composition at a given E-field and furnace temperature, better controls can be implemented to improve sintering behaviour through controlled FS. These could include limiting currents, fixing furnace temperature and applying the required E-field to the given composition, applying the E-field in different steps during heating or at a fixed temperature etc. Likewise, cooling programs may be optimised to prevent cracking of the sample. Once uniformly dense samples are achieved, more details can be extracted from the effect of flash sintering on the microstructure. The extremely fast sintering cycles can also effect yttria distribution. If the starting material has a uniform yttria distribution across the grains, the short sintering treatments and temperatures reached may not give sufficient time for segregation to occur and even grains with larger grain sizes can be made resistant to HTA.

\section{Conclusions:}

The electrical characterization of various zirconia-toughened alumina (ZTA) compositions were examined under different E-fields to understand the relationship between ZTA composition, applied E-field, and the onset temperature of flash sintering. It was found that below $10 \mathrm{wt} \%$ alumina, the flash behaviour remains the same as the pure 3YSZ phase. Above this content, the onset of flash sintering temperature begins to increase with the increase in alumina content. With the setup used, $300 \mathrm{~V} / \mathrm{cm}$ was able to cause flash sintering in $30 \mathrm{ZTA}$ at $1000^{\circ} \mathrm{C}$. Regardless of the ZTA composition, the onset of flash sintering occurs at a critical volumetric power dissipation of $0.02 \mathrm{~W} / \mathrm{mm}^{3}$. 
Fixing the composition at 25ZTA, at $300 \mathrm{~V} / \mathrm{cm}$ flash was not achieved due to electrode

2 limitations, but by increasing the E-field to $500 \mathrm{~V} / \mathrm{cm}$ and to $700 \mathrm{~V} / \mathrm{cm}$ flash sintering was 3 possible. The flash sintering onset temperature with $700 \mathrm{~V} / \mathrm{cm}$, the highest E-field used, was 4 just $918{ }^{\circ} \mathrm{C}$, around $600{ }^{\circ} \mathrm{C}$ lower than conventional sintering needs.

5 A variety of microstructures were found in 25ZTA sintered with $700 \mathrm{~V} / \mathrm{cm}$. Areas where 6 under-sintering had occurred were found at the edges of the material farthest from the starting 7 of sintering, and melted regions with large amounts of grain growth were found in the area 8 where sintering was localized. However, in the bulk of the sample, full dense microstructures 9 that had no evident grain growth were achieved. Zirconia grain sizes lower than $100 \mathrm{~nm}$ were 10 maintained suggesting that the material should have full HTA resistance in those regions.

11 Better control of the current and sintering conditions can be implemented further to solve the issues of localized melting and under-sintering to achieve fully dense nanostructured ZTA 13 composites, thus paving the way for fabricating engineering components for demanding 14 applications.

16 Acknowledgements:

17 The authors thank the Engineering and Physical Sciences Research Council (EPSRC), UK 18 (Nanoplants grant: EP/L024780/1) and Innovate UK for financial support. 
1. De Aza, A. H., Chevalier, J., Fantozzi, G., Schehl, M. \& Torrecillas, R. Crack growth resistance of alumina, zirconia and zirconia toughened alumina ceramics for joint prostheses. Biomaterials 23, 937-945 (2002).

2. Piconi, C. \& Maccauro, G. Zirconia as a ceramic biomaterial. Biomaterials 20, 1-25 (1999).

3. Administration, U. S. F. and D. St. Gobain Desmarquest Zirconia Ceramic Femoral Head. (2013). at <http://www.fda.gov/Safety/MedWatch/SafetyInformation/SafetyAlertsforHumanMedi calProducts/ucm172706.htm>

4. $\quad$ Reece, M. \& Guiu, F. Indentation Fatigue of High-Purity Alumina in Fluid Environments. J. Am. Ceram. Soc. 74, 148-154 (1991).

5. Hydrothermal Reactions for Materials Science and Engineering. (Springer Netherlands, 1990). doi:10.1007/978-94-009-0743-0

6. Gupta, T. K., Bechtold, J. H., Kuznicki, R. C., Cadoff, L. H. \& Rossing, B. R. Stabilization of tetragonal phase in polycrystalline zirconia. J. Mater. Sci. 12, 24212426 (1977).

7. Paul, A., Vaidhyanathan, B. \& Binner, J. G. P. Hydrothermal Aging Behavior of Nanocrystalline Y-TZP Ceramics. J. Am. Ceram. Soc. 94, 2146-2152 (2011).

8. Chevalier, J., Gremillard, L. \& Deville, S. Low-Temperature Degradation of Zirconia and Implications for Biomedical Implants. Annu. Rev. Mater. Res. 37, 1-32 (2007).

9. Binner, J. \& Vaidhyanathan, B. Processing of bulk nanostructured ceramics. J. Eur. Ceram. Soc. 28, 1329-1339 (2008).

10. Vaidhyanathan, B. \& Binner, J. G. P. Novel Processing of Nanostructured Ceramics using Microwaves. in AIChE Annual Meeting 1419 (2004).

11. Eichler, J., Rödel, J., Eisele, U. \& Hoffman, M. Effect of Grain Size on Mechanical Properties of Submicrometer 3Y-TZP: Fracture Strength and Hydrothermal Degradation. J. Am. Ceram. Soc. 90, 2830-2836 (2007).

12. Muñoz-Saldaña, J., Balmori-Ramírez, H., Jaramillo-Vigueras, D., Iga, T. \& Schneider, 
G. A. Mechanical properties and low-temperature aging of tetragonal zirconia polycrystals processed by hot isostatic pressing. J. Mater. Res. 18, 2415-2426 (2011).

13. Guicciardi, S., Shimozono, T. \& Pezzotti, G. Ageing effects on the nanoindentation response of sub-micrometric 3Y-TZP ceramics. J. Mater. Sci. 42, 718-722 (2007).

14. Orrù, R., Licheri, R., Locci, A. M., Cincotti, A. \& Cao, G. Consolidation/synthesis of materials by electric current activated/assisted sintering. Mater. Sci. Eng. R Reports 63, 127-287 (2009).

15. Menezes, R. R. \& Kiminami, R. H. G. a. Microwave sintering of alumina-zirconia nanocomposites. J. Mater. Process. Technol. 203, 513-517 (2008).

16. Binner, J., Vaidhyanathan, B., Paul, A., Annaporani, K. \& Raghupathy, B. Compositional Effects in Nanostructured Yttria Partially Stabilized Zirconia. Int. J. Appl. Ceram. Technol. 8, 766-782 (2011).

17. Cologna, M., Rashkova, B. \& Raj, R. Flash Sintering of Nanograin Zirconia in $5 \mathrm{~s}$ at 850․ J. Am. Ceram. Soc. 93, 3556-3559 (2010).

18. Cologna, M., Francis, J. S. C. \& Raj, R. Field assisted and flash sintering of alumina and its relationship to conductivity and MgO-doping. J. Eur. Ceram. Soc. 31, 28272837 (2011).

19. Naik, K., Sglavo, V. \& Raj, R. Field assisted sintering of ceramic constituted by alumina and yttria stabilized zirconia. J. Eur. Ceram. Soc. 34, 2435-2442 (2014). 
2

3 Table I: ZTA samples prepared for flash sintering with wt\% ratios and the equivalent vol\% 4 for the 3YSZ content.

\begin{tabular}{llll} 
Composition & wt\% 3YSZ & wt\% alumina & Vol\% 3YSZ \\
\hline 3YSZ & 100 & 0 & 100 \\
90ZTA & 90 & 10 & 85.6 \\
80ZTA & 80 & 20 & 72.5 \\
30ZTA & 30 & 70 & 22.0 \\
25ZTA & 25 & 75 & 18.0
\end{tabular}

5

6 


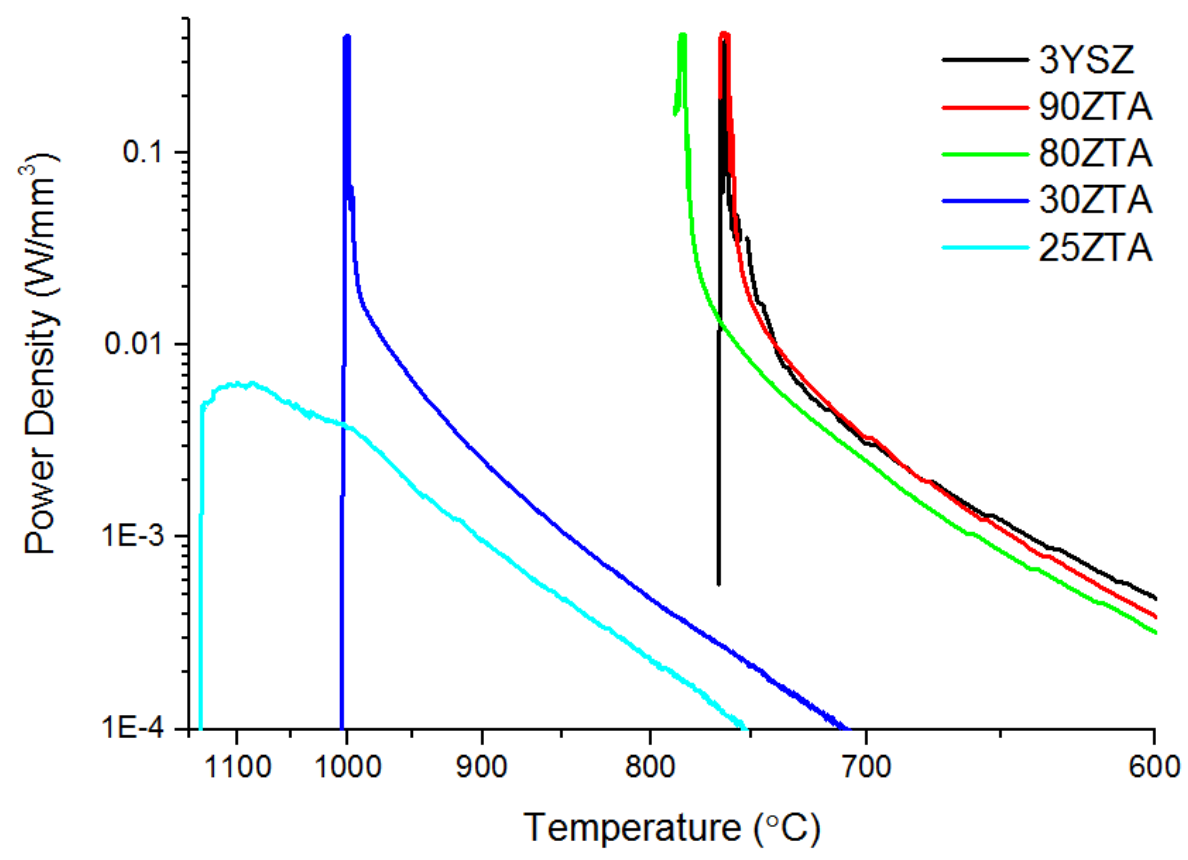

2

3

4 Figure 1: The power dissipation of the flash sintering behaviour for the range of ZTA 5 compositions with an E-field of $300 \mathrm{~V} / \mathrm{cm}$

6

7 


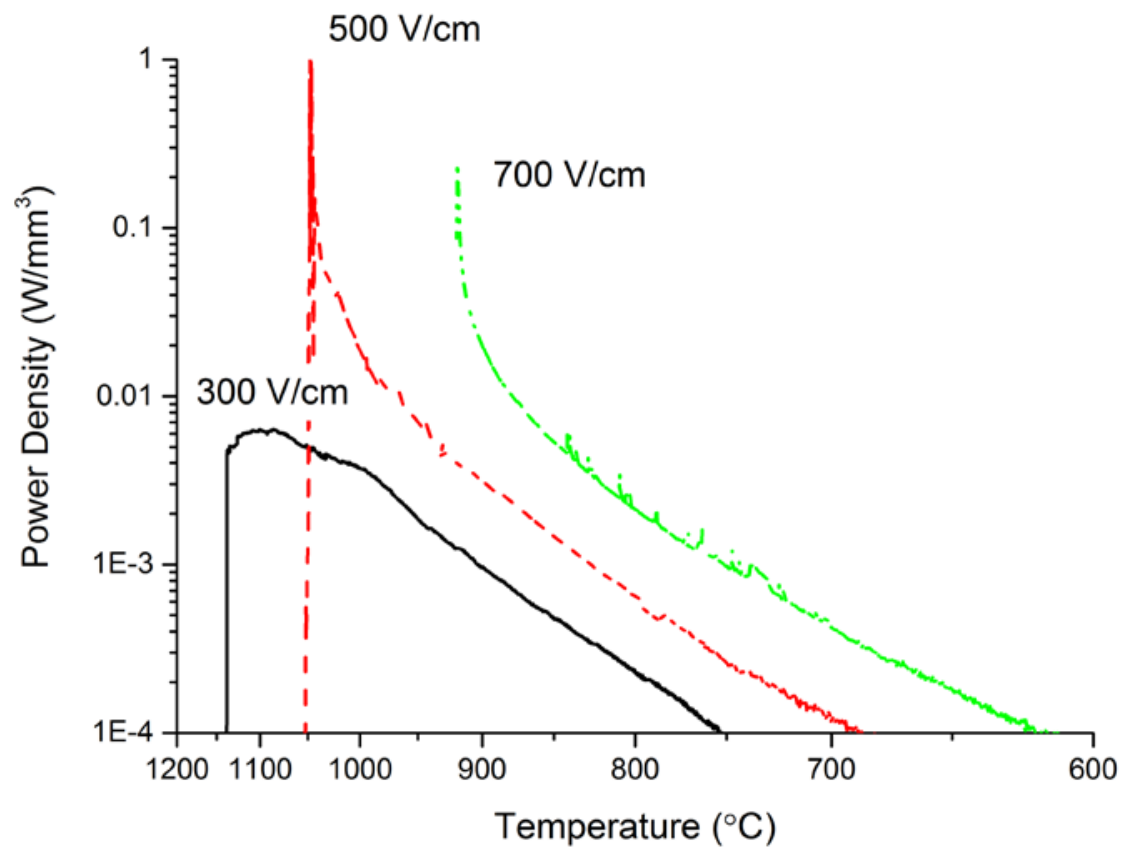

1

2 Figure 2: Power dissipation behaviour for flash sintering of 25ZTA under different applied E3 fields

4

5 


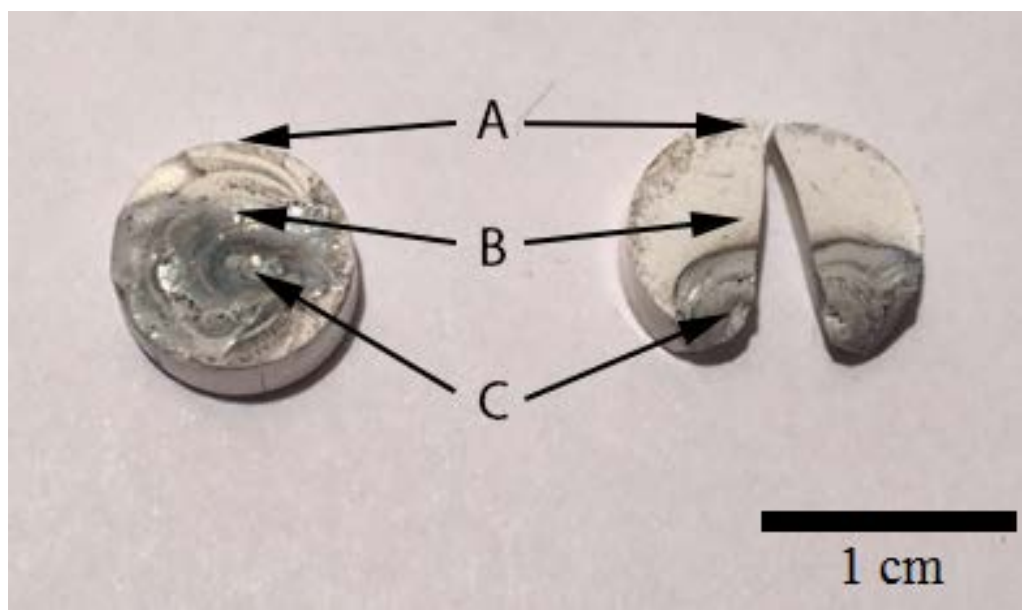

2

3 Figure 3: Examples of flash sintered 25 ZTA sintered with $700 \mathrm{~V} / \mathrm{cm}$ with A) under-sintered 4 regions $\mathrm{B}$ ) well sintered regions, and C) melted regions

5

6

7

8 
